\title{
Synergy of understanding dermatologic disease and epidermal biology
}

\author{
John R. Stanley \\ University of Pennsylvania School of Medicine, Department of Dermatology, Philadelphia, Pennsylvania, USA.
}

\begin{abstract}
Dermatologic disease, although seldom life threatening, can be extremely disfiguring and interfere with the quality of life. In addition, as opposed to other organs, just the aging of skin and its adnexal structure the hair follicle can result in cosmetic concerns that affect most of us. The articles in this dermatology Review Series demonstrate recent progress in understanding the cell biology and molecular pathophysiology of the epidermis and hair follicles, which harbor keratinocyte and melanocyte stem cells. They reveal a dynamic relationship between research and clinical care: knowledge of dermatologic disease has facilitated the understanding of the biology of the epidermis and, in turn, progress in basic science has informed our understanding of disease. This type of synergy is a profound strength of clinical research of the type that the JCI is dedicated to publishing.
\end{abstract}

\section{Introduction}

The Reviews in this series highlight the progress in understanding several dermatologic diseases that are particularly important because they are common and/or disfiguring. Kubo et al. (1) highlight recent major progress in understanding the pathophysiology of atopic dermatitis (Figure 1), a disease both very common (estimates are $10 \%-20 \%$ prevalence in children; ref. 2) and potentially severe and difficult to deal with for both patients and parents. Myung and Ito (3) discuss recent progress in understanding the bulge of the hair follicle and its importance in harboring both keratinocyte and melanocyte stem cells. Understanding the cell biology of the bulge is important for dissecting the pathophysiology of skin cancer-like basal cell carcinoma (BCC) as well as the potentially disfiguring disease vitiligo (Figure 2 ). In addition, the melanocyte stem cell contributes to hair pigment. Insights into this stem cell may help us understand the physiologic process of the graying of hair with age. Normal graying of hair, although not a disease, is of great concern to millions of people, probably because pigment in hair is an indication of youth (Figure 3 ). Finally, Kasper et al. (4) and Ratushny et al. (5) discuss new aspects of the pathophysiology of skin cancers. Not only are cancers of the skin some of the most common cancers in humans, but they can be terribly disfiguring if left untreated (Figure 4).

\section{Atopic dermatitis and the epidermal barrier}

On clinical grounds alone, atopic dermatitis has been a difficult disease to understand. There is no primary skin lesion (e.g., a blister or papule) and no pathognomonic histology, issues that make mouse models of disease problematic. In general, the diagnosis is based on a constellation of findings such as the distribution of lesions, presence of pruritus, lichenification (thickening of the epidermis with accentuated skin markings) secondary to scratching, susceptibility to staphylococcal infection or colonization, associated atopic diseases such as allergic rhinitis or asthma, and certain immunologic abnormalities (increased serum IgE and defects in viral cell-mediated immunity). Recent groundbreaking work on the genetics of atopic dermatitis (6) focused our attention on the epidermal barrier in this disease

Conflict of interest: The author has declared that no conflict of interest exists. Citation for this article: J Clin Invest. 2012;122(2):436-439. doi:10.1172/JCI62237. as well as other related diseases and gave impetus to the study of the epidermal barrier in immunology. This study, subsequently confirmed by many others, showed that atopic dermatitis was strongly associated with common mutations in the gene encoding filaggrin, a protein produced in the superficial epidermis that is thought to be important in maintaining its barrier function. Interestingly, another disease, ichthyosis vulgaris, suggested that filaggrin might be an important molecule in atopic dermatitis (7). Patients with this disease of scaly skin often have atopic dermatitis, and their histology often shows a lack of keratohyalin granules, which contain filaggrin. The genetic studies in atopic dermatitis also showed a high correlation of filaggrin mutations with atopic dermatitis-associated asthma, but no correlation with asthma not associated with atopic dermatitis. Other diseases of the epidermal barrier are also associated with atopic dermatitis. One example is Netherton syndrome, an autosomal recessive disease in which patients have ichthyosis with atopic dermatitis, allergic rhinitis, increased serum IgE, and a barrier defect (8). Genetic analysis of these patients indicates a mutation in the SPINK5 gene that encodes LEKTI, a serine protease inhibitor in the superficial epidermis. Patients who lack this inhibitor have excess protease activity that degrades filaggrin, among other molecules (9), resulting in a barrier defect and atopy.

In response to these novel insights, many researchers have turned their focus to understanding the epidermal barrier, highlighted in this series by Kubo et al. (1). This barrier is not a simple barrier against water loss, but consists of at least three components. First is a liquid-liquid barrier formed by tight junctions in the epidermis. These junctions prevent paracellular diffusion of liquids around the cells of the epidermis and are located in the stratum granulosum (cells in the superficial living epidermis that contain keratohyalin granules). The second barrier is formed by the stratum corneum at the air-liquid interface. This barrier contains keratin organized by filaggrin into compact flat sheets and contains the lipid contents of lamellar granules. This barrier presumably prevents evaporation of water from the skin surface, holds moisture in the stratum corneum, and helps block antigen access to the immune system. Finally, Langerhans cells form an immune barrier. Under conditions of stress, these cells can be activated and their dendrites extended beyond the tight junction barrier to process antigen for Th2 responses $(10,11)$. 


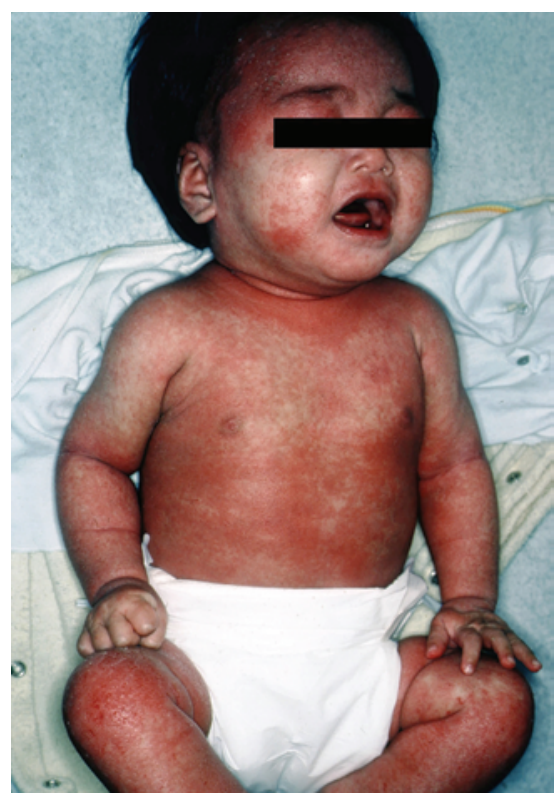

\section{Figure 1}

Child with severe atopic dermatitis. Such disease profoundly affects the quality of life of both the child and parents. Reproduced with permission from William James.

Precisely how these types of barriers, and perhaps others, contribute to antigen processing and diseases such as atopic dermatitis and atopic asthma remains to be determined, but presumably percutaneous antigen processing is intimately involved. However, present knowledge suggests that trying to maintain an intact epidermal barrier from infancy might decrease the development of atopic dermatitis.

\section{Scarring alopecias, vitiligo, gray hair, and hair follicle stem cells}

Myung and Ito (3) review the current understanding of where epithelial and melanocyte stem cells are located in the hair follicle. Many years of research have suggested that epithelial stem cells are present in the bulge, which is at the base of the telogen-phase (resting) hair follicle and near the arrector pili insertion of the anagen-phase (growing) hair follicle (12). The bulge is sometimes a theoretical anatomical area (i.e., it is not clearly apparent as a bulge anatomically), but staining of epithelial cells for stem cell markers can clearly identify their location within the hair follicle. Epithelial stem cells from the bulge area can give rise to all layers of the hair follicle each time it is regenerated from the short telogen follicle to the long anagen follicle that actively grows a hair shaft. Without the stem cells in the bulge, the hair follicle will not regenerate (i.e., there is permanent hair loss), with the unusual exception of neogenesis of the hair follicle in mouse wounds (13). Initially it was believed that stem cells give rise to transient amplifying cells, which are then committed to produce the hair follicle. However, more detailed studies discussed in the review by Myung et al. (3) have recently suggested that stem cells may give rise to a secondary hair germ (sometimes anatomically apparent beneath the bulge in telogen hair), and that this secondary hair germ may not only give rise to cells committed to form the hair follicle but also to cells that may "reverse" to regenerate stem cells in the bulge.
Hair pigment comes from melanocytes in the hair matrix (which contains the rapidly proliferating epithelial cells that produce the hair shaft). However, these melanocytes die with the end of each hair cycle, and new melanocytes develop from melanocyte stem cells with each new anagen cycle. These melanocyte stem cells are also in the bulge, and recent studies have shown some of the characteristic niche requirements to maintain and activate them (14).

Various observations in disease and aging complement the above findings. For example, the importance of the keratinocyte stem cells in the bulge is underscored by the pathophysiology of scarring alopecia (loss of hair that does not grow back), in which the bulge is targeted by the immune system and destroyed. One very typical disease in humans resulting in scarring alopecia is lichen planopilaris. In this disease a $\mathrm{T}$ cell infiltrate targets the bulge and causes apoptosis of keratinocytes (15). Cutaneous lupus erythematosus is another inflammatory disease that causes disfiguring scarring alopecia. In these diseases, the loss of the keratinocyte stem cell is the cause of the inability to grow new hair. An example of the importance of the melanocyte stem cell is illustrated by the graying of hair with age and the disease vitiligo. Hair color is a sign of youth (Figure 3), and most elderly people lose their hair pigment (but, interestingly, not skin pigment). This physiologic loss of hair pigment is thought to be due to loss of follicular melanocyte stem cells (16). Finally, it is known that in vitiligo, in which an autoimmune attack on melanocytes in epidermis causes their loss, repigmentation after therapy often derives from a reservoir of melanocyte precursors in the hair follicle, presumably from stem cells in the bulge $(17,18)$.

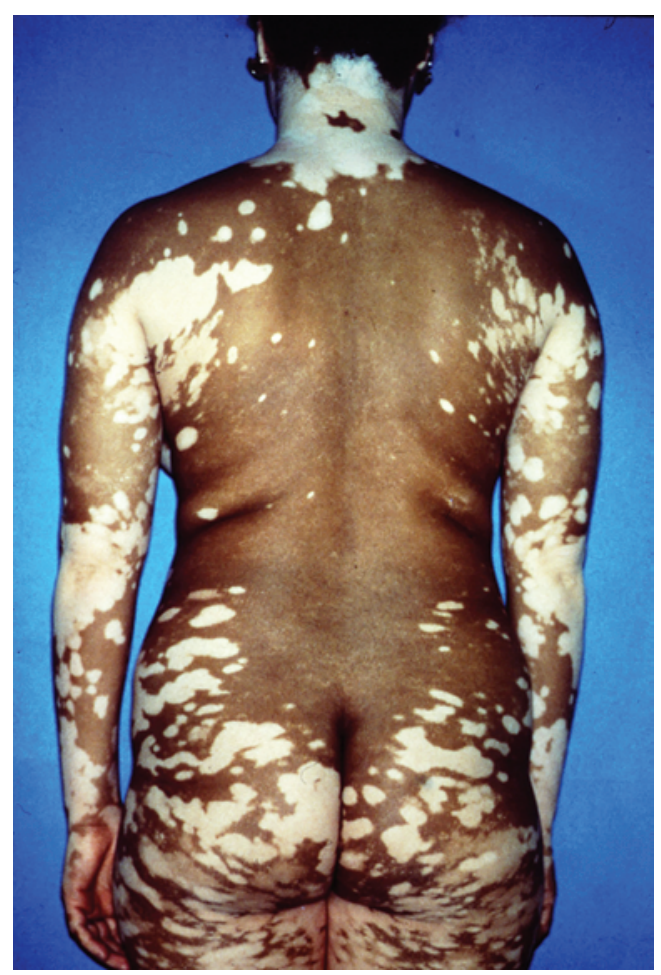

\section{Figure 2}

Patient with vitiligo. This depigmenting disease is particularly disfiguring in dark-skinned patients. Reproduced with permission from William James. 


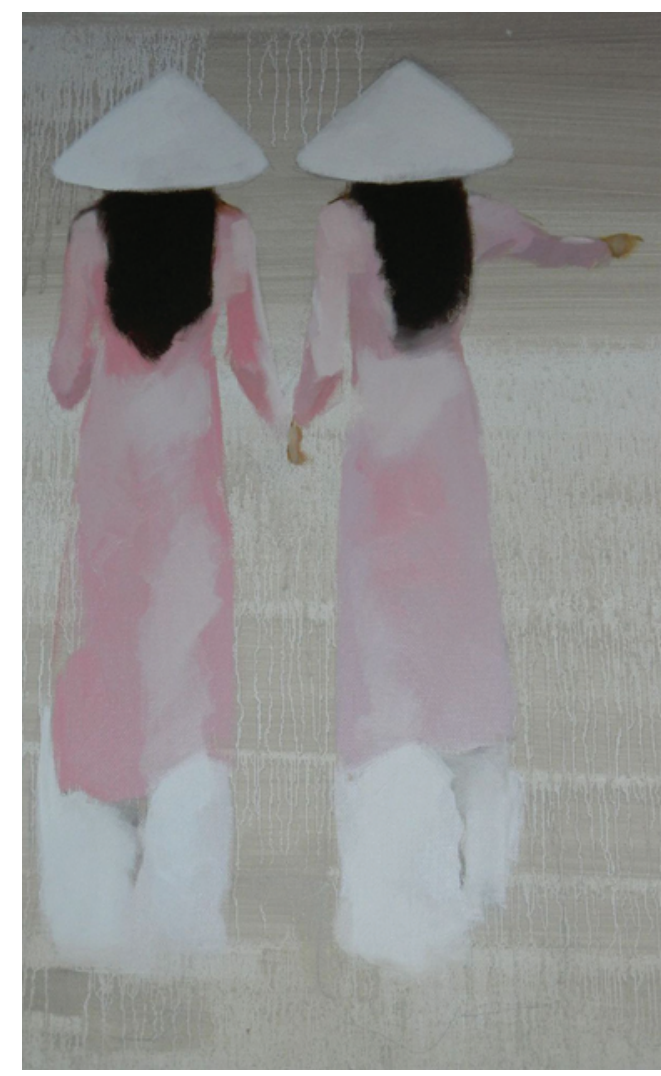

Figure 3

Young Vietnamese women (detail from Schoolgirls, a painting by Nguyen Vinh Binh). Their black hair indicates that these women are young, not old.

There are important clinical (and cosmetic) implications of the above findings. For example, if we want to permanently eliminate unwanted hair in humans (a common clinical problem), we will have to eliminate the bulge, not just the matrix. Currently, electrolysis or laser hair removal does not always do this, and therefore hair often regrows. To prevent permanent hair loss in lupus or other diseases, the stem cell in the bulge must be protected from destruction. To maintain hair color, we will have to understand what niche factors in the bulge keep the melanocyte stem cell alive. Finally, to replace hair pigment for patients with vitiligo, we will have to protect the melanocyte stem cell and learn how to reactivate it to repopulate the epidermis. These few examples indicate the importance of understanding the anatomically subtle, but biologically critical, bulge of the hair follicle.

\section{Epidermal carcinogenesis: insights from human syndromes and mouse models}

A basic mouse model of skin carcinogenesis is the multistage model involving initiation, promotion, and progression (19). From this model we have learned a tremendous amount about carcinogenesis. However, this disease, in which papillomas give rise to squamous cell carcinoma (SCC), does not really look like the most prevalent types of skin cancer in humans. In humans, skin cancer or its precursor is mostly either BCC or actinic keratosis, which is thought to be a precursor to the most common types of SCC.
BCC is the most common cancer in humans and has not, until recently, been seen in mouse models of carcinogenesis (20). Genetic analysis of basal cell nevus syndrome, in which patients develop hundreds of BCCs starting at a very early age, demonstrated that these patients carried mutations in the Patched 1 (PTCH1) gene (21). Loss of PTCH1 protein activates Hedgehog signaling. Furthermore, most sporadic BCCs in elderly patients have activating mutations in this pathway (22). By genetically activating this pathway in different areas of the mouse epidermis and hair follicle, characteristic types (e.g., nodular, superficial) of BCCs can be produced, thus establishing mouse models that more closely mimic human BCC (23-27). As reviewed in Kasper et al. in this review series (4), the analysis of the critical importance of the Hedgehog pathway in BCC and of mouse models in which this pathway is activated in various cells and under various conditions (e.g., wounding) have led to new understandings of the molecular genetics and cell biology of this cancer as well as to new systemic and therapeutic approaches to target the Hedgehog pathway for patients with BCCs that are difficult to control.

SCC of the skin is the second most common cancer in humans, and its precursor lesion, actinic keratosis, is even more common. As discussed in the Review by Ratushny et al. (5) in this series, SCC is much more genetically complicated than BCC. Although p53 mutation is probably very important in SCC development and progression, many other gene pathways have been implicated, some of which suppress p53 and some of which seem to act independently. These observations have given rise to the idea that SCC may arise by a classic multistep carcinogenesis model, in which a precursor lesion (or lesions), due to genetic instability, accrues additional mutations that drive disease progression. However, because few mouse models actually demonstrate obvious actinic keratosis-like precursors before the development of SCC, the progression of this cancer, as relevant to humans, has been difficult to study. Recently, however, a transgenic mouse bearing an activated allele of the tyrosine kinase Fyn has been developed that shows typical-looking (both clinically and histologically) actinic keratosis that progresses to later-stage SCCs (28). This model should prove useful not only for studying

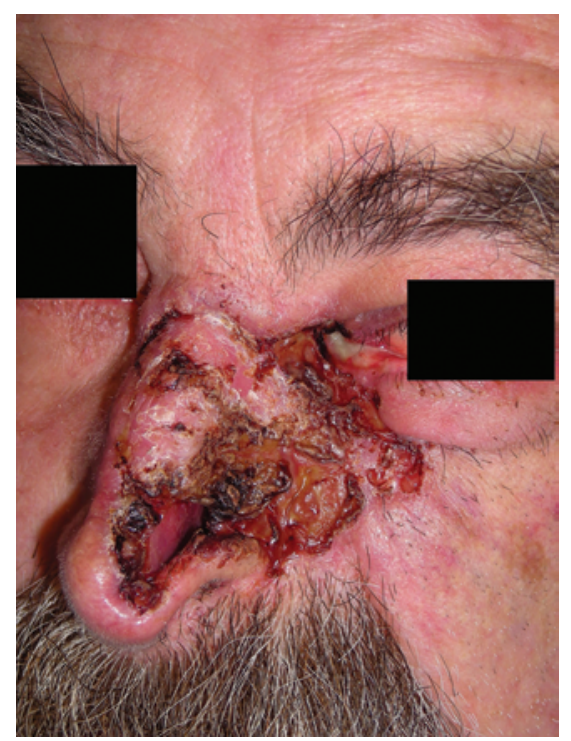

Figure 4

Destructive BCC. Reproduced with permission from Christopher Miller. 
the genetic pathophysiology of development of some human SCCs but also for the study of topical agents to prevent that progression.

\section{Conclusion}

The reviews in this dermatology Review Series illustrate, in a cross-section of dermatologic diseases of the epidermis, the importance of the synergy of understanding of both human disease and basic biology to further our understanding of both in skin and its diseases. This type of synergy is representative of a basic paradigm of science that underlies much of the science that the JCI encourages in its publication.

\section{Acknowledgments}

This work was supported by grant P30-AR-057217 (to the Penn Skin Diseases Research Center) from the National Institute of Arthritis and Musculoskeletal and Skin Diseases.

Address correspondence to: John R. Stanley, Department of Dermatology, 211B Clinical Research Bldg, University Of Pennsylvania, 415 Curie Blvd, Philadelphia, Pennsylvania 19104, USA. Phone: 215.898.3240; Fax: 215.573.2033; E-mail: jrstan@ mail.med.upenn.edu.
1. Kubo A, Nagao K, Amagai M. Epidermal barrier dysfunction and cutaneous sensitization in atopic diseases. J Clin Invest. 2012;122(2):440-447.

2. Leung DW, Eichenfield LF, Boguniewicz M. Atopic dermatitis. In: Wolff K, Goldsmith LA, Katz SI, Gilchrest BA, Paller AS, Leffell DJ, eds. Fitzpatrick's Dermatology in General Medicine. 7th ed. New York, New York, USA: McGraw-Hill; 2008:146-158.

3. Myung P, Ito M. Dissecting the bulge in hair regeneration. J Clin Invest. 2012;122(2):448-454.

4. Kasper M, Jaks V, Hohl D, Toftgård R. Basal cell carcinoma - molecular biology and potential new therapies. J Clin Invest. 2012;122(2):455-463.

5. Ratushny V, Gober MD, Hick R, Ridky TW, Seykora JT. From keratinocyte to cancer: the pathogenesis and modeling of cutaenous squamous cell carcinoma. J Clin Invest. 2012;122(2):464-472.

6. Palmer $\mathrm{CN}$, et al. Common loss-of-function variants of the epidermal barrier protein filaggrin are a major predisposing factor for atopic dermatitis. Nat Genet. 2006;38(4):441-446.

7. Hudson TJ. Skin barrier function and allergic risk Nat Genet. 2006;38(4):399-400.

8. Chavanas S, et al. Mutations in SPINK5, encoding a serine protease inhibitor, cause Netherton syndrome. Nat Genet. 2000;25(2):141-142.

9. Bonnart $\mathrm{C}$, et al. Elastase 2 is expressed in human and mouse epidermis and impairs skin barrier function in Netherton syndrome through filaggrin and lipid misprocessing. J Clin Invest. 2010; 120(3):871-882.

10. Kubo A, Nagao K, Yokouchi M, Sasaki H, Amagai M. External antigen uptake by Langerhans cells with reorganization of epidermal tight junction barriers. J Exp Med. 2009;206(13):2937-2946.

11. Ouchi $\mathrm{T}$, et al. Langerhans cell antigen capture through tight junctions confers pre-emptive immunity in experimental staphylococcal scalded skin syndrome. J Exp Med. 2011;208(13):2607-2613.

12. Cotsarelis G, Sun TT, Lavker RM. Label-retaining cells reside in the bulge area of pilosebaceous unit: implications for follicular stem cells, hair cycle, and skin carcinogenesis. Cell. 1990;61(7):1329-1337.

13. Ito $\mathrm{M}$, et al. Wnt-dependent de novo hair follicle regeneration in adult mouse skin after wounding. Nature. 2007;447(7142):316-320.

14. Rabbani $P$, et al. Coordinated activation of Wnt in epithelial and melanocyte stem cells initiates pigmented hair regeneration. Cell. 2011;145(6):941-955.

15. Mobini N, Tam S, Kamino H. Possible role of the bulge region in the pathogenesis of inflammatory scarring alopecia: lichen planopilaris as the prototype. J Cutan Pathol. 2005;32(10):675-679.

16. Nishimura EK, Granter SR, Fisher DE. Mechanisms of hair graying: incomplete melanocyte stem cell maintenance in the niche. Science. 2005; 307(5710):720-724.

17. Staricco RG, Miller-Milinska A. Activation of the amelanotic melanocytes in the outer root sheath of the hair follicle following ultra violet rays exposure. J Invest Dermatol. 1962;39:163-164.

18. Ortonne JP, Schmitt D, Thivolet J. PUVA-induced repigmentation of vitiligo: scanning electron microscopy of hair follicles. J Invest Dermatol. 1980; 74(1):40-42.

19. Yuspa SH, et al. Role of oncogenes and tumor suppressor genes in multistage carcinogenesis. J Invest Dermatol. 1994;103(5 suppl):90S-95S.
20. Carucci JA, Leffell DJ. Basal cell carcinoma. In: Wolff K, Goldsmith LA, Katz SI, Gilchrest BA, Paller AS, Leffell DJ, eds. Fitzpatrick's Dermatology in General Medicine. 7th ed. New York, New York, USA: McGraw-Hill; 2008:1036-1042.

21. Oro AE. Basal cell nevus syndrome. In: Wolff K, Goldsmith LA, Katz SI, Gilchrest BA, Paller AS, Leffell DJ, eds. Fitzpatrick's Dermatology in General Medicine. 7th ed. New York, New York, USA: McGraw-Hill; 2008:1042-1048.

22. Xie J, et al. Activating Smoothened mutations in sporadic basal-cell carcinoma. Nature. 1998; 391(6662):90-92.

23. Youssef KK, et al. Identification of the cell lineage at the origin of basal cell carcinoma. Nat Cell Biol. 2010;12(3):299-305.

24. Johnson RL, et al. Human homolog of patched, a candidate gene for the basal cell nevus syndrome. Science. 1996;272(5268):1668-1671.

25. Aszterbaum $M$, et al. Ultraviolet and ionizing radiation enhance the growth of BCCs and trichoblastomas in patched heterozygous knockout mice. Nat Med. 1999;5(11):1285-1291.

26. Grachtchouk $M$, et al. Basal cell carcinomas in mice arise from hair follicle stem cells and multiple epithelial progenitor populations. J Clin Invest. 2011;121(5):1768-1781.

27. Kasper M, et al. Wounding enhances epidermal tumorigenesis by recruiting hair follicle keratinocytes. Proc Natl Acad Sci U S A. 2011; 108(10):4099-4104.

28. Zhao L, et al. Srcasm inhibits Fyn-induced cutaneous carcinogenesis with modulation of Notch1 and p53. Cancer Res. 2009;69(24):9439-9447. 